\title{
Globe
}

Revue internationale d'études québécoises

\section{Enjeux de la coexistence}

\section{Pierre Coulombe}

Volume 2, numéro 2, 1999

Les enjeux de la coexistence linguistique

URI : https://id.erudit.org/iderudit/1000466ar

DOI : https://doi.org/10.7202/1000466ar

Aller au sommaire du numéro

Éditeur(s)

Globe, Revue internationale d'études québécoises

ISSN

1481-5869 (imprimé)

1923-8231 (numérique)

Découvrir la revue

Citer ce document

Coulombe, P. (1999). Enjeux de la coexistence. Globe, 2(2), 9-12.

https://doi.org/10.7202/1000466ar d'utilisation que vous pouvez consulter en ligne.

https://apropos.erudit.org/fr/usagers/politique-dutilisation/ 


\title{
Introduction Enjeux de la coexistence
}

\author{
Pierre Coulombe \\ Université McGill
}

Les expériences canadienne et québécoise en matière de relations intercommunautaires sont, on le sait, riches en enseignement. Qu'il s'agisse des groupes ethnolinguistiques (francophone, anglophone, allophone) au sein même du Québec, ou des communautés nationales qui composent la fédération canadienne (québécoise, autochtones), les enjeux de la coexistence sont importants. Non seulement renvoient-ils à la coexistence d'identités de toutes sortes, mais aussi à la coexistence de visions politiques qui parfois divergent, parfois convergent, quant à la meilleure façon de gérer cette pluralité. Les politiques linguistiques en sont un exemple éloquent : la rencontre entre les orientations fédérale et québécoise dans l'aménagement linguistique a engendré une dynamique idéologique et politique complexe à l'aube du XXIe siècle, dynamique que certains auteurs chercheront d'ailleurs à mieux comprendre dans les articles qui suivent.

Le dossier s'ouvre sur un article de James Tully où il traite de reconnaissance, non pas tant comme un produit constitutionnel, mais comme un processus, une activité politique continue et fluide au cours de laquelle les identités à reconnaître sont elles-mêmes changeantes. Puisque toute règle de reconnaissance implique paradoxalement un déni de reconnaissance, l'auteur s'interroge sur la forme de société pluraliste et plurinationale qui procurerait à ses membres la liberté de débattre et éventuellement d'amender les règles du jeu constitutionnel de la reconnaissance. Pour lui, une telle société serait respectueuse du droit à l'autodétermination de ses

Pierre Coulombe, «Enjeux de la coexistence*, Globe. Revue internationale d'études québécoises, vol. 2, no 2, 1999. 


\section{REVUE INTERNATIONALE D'ÉTUDES QUÉBÉCOISES}

composantes et accueillerait l'idée d'un dialogue permanent entre elles. Or, selon l'auteur, la pratique constitutionnelle dominante au Canada et les formules d'amendement qui l'encadrent ne répondent pas à cette exigence. La Cour suprême du Canada, dans son avis sur le droit de sécession du Québec, offrirait néanmoins une piste à suivre dans la mesure où l'obligation de dialoguer qu'elle prescrit s'inscrit dans la vision d'une société libre en tant que système équitable de coopération.

Si les gouvernements canadien et québécois célèbrent depuis une quarantaine d'années la diversité linguistique, selon Angéline Martel le modèle stato-national des rapports de pouvoirs dans lequel ils s'inscrivent les empêchent de gérer adéquatement les problèmes qu'elle leur apporte. Ce sont les fondements mêmes de l'Etat qu'elle met en cause, ses pratiques et ses cadres régulateurs qui relèvent davantage d'une stratégie de pouvoir que d'une relation de partage. Tant les politiques linguistiques du Québec que celles du Canada souffrent, selon elle, d'une idéologie qui empêche de lever les nombreux défis de l'aménagement de la diversité linguistique.

Jean Dansereau, pour sa part, fait le point sur la Charte de la langue française. Il fait d'abord remarquer que puisque la Charte de la langue française vise à faire du français la langue de la société civile au Québec, alors que la politique fédérale de bilinguisme officiel prévoit surtout l'égalité d'accès des francophones et anglophones du pays à des services gouvernementaux, on ne peut parler a priori d'incompatibilité entre les deux. L'auteur passe ensuite en revue les arrêts de la Cour suprême touchant la Loi 101, notamment l'arrêt Ford sur la langue d'affichage qui, ayant reconnu la légitimité des objectifs poursuivis par la Charte de la langue française et les éléments d'asymétrie qui en découlent, pourrait venir valider d'autres composantes de la politique linguistique du Québec. Il conclut que si les aspects essentiels de la Charte de la langue française sont demeurés intacts face aux jugements des tribunaux, il faut néanmoins continuer d'affirmer sa légitimité face à ceux qui véhiculent l'idée qu'elle a été charcutée au point de succomber. 


\section{LA POLITIQUE LINGUISTIQUE DU QUÉBEC}

Contrairement à ceux qui affirment que la politique linguistique fédérale nuit aux efforts du Québec de faire du français la langue commune, Michael MacMillan soutient qu'ils sont tout à fait compatibles. Retraçant l'évolution des avancées et des reculs des deux politiques au fil des ans, l'auteur constate que les différences entre elles se sont dans les faits atténuées. Pour lui, la langue française possède aujourd'hui tous les attributs nécessaires à sa survie en territoire québécois, et si la politique fédérale de bilinguisme officiel vient épauler la communauté anglo-québécoise, elle ne menace pas pour autant le statut du français. Il est donc fort probable que la forme de ces deux politiques demeurera inchangée dans le moyen terme.

Ines Molinaro s'intéresse quant à elle aux politiques d'intégration des États canadien et québécois à l'égard des communautés allophones, politiques qui s'inspirent à la fois des normes de valorisation de la diversité culturelle et des impératifs liés à la construction d'une communauté nationale. Ces politiques reflètent le désir de réaliser une citoyenneté commune qui transcenderait les différences ethnolinguistiques, mais, selon l'auteure, les contextes historiques dans lesquels elles se sont déployées engendrent néanmoins des formes d'exclusion. Son analyse porte surtout sur les défis que pose le modèle québécois, notamment sur sa capacité de bâtir une culture civique francophone qui inclut comme membres à part entière les immigrants allophones.

Dans une perspective comparée, Luisa Domenichelli discute de la capacité des constitutions des États plurinationaux, tels que la Belgique et le Canada, de traduire en principes juridiques les diverses valeurs des communautés qui les composent et de servir d'instruments de médiation des relations intercommunautaires. Selon elle, l'hétérogénéité de la Constitution belge permet aux tribunaux de tenir compte des attentes en apparence irréconciliables des diverses composantes et de trouver les compromis nécessaires. Elle soutient que la Constitution canadienne, à l'opposé, est partiale puisque le Québec n'y trouve pas la reconnaissance de ses valeurs et, par conséquent, que l'ordre juridique canadien ne favorise pas la conciliation des intérêts des composantes de la fédération. 


\section{REVUE INTERNATIONALE D'ÉTUDES QUÉBÉCOISES}

En somme, la coexistence de communautés ethnolinguistiques et nationales au sein des sociétés québécoise et canadienne pose des défis particuliers dans la recherche de la justice et de la stabilité. L'architecture politico-légale qui en résulte et la grammaire morale qui la sous-tend font l'objet de ce dossier. 